\title{
RESEARCH
}

\section{Completely multiplicative functions with sum zero over generalised prime systems}

Ammar Ali Neamah

\author{
*Correspondence: \\ a.a.n.al-rammahi@pgr.reading.ac.uk; \\ ammara.meamah@uokufa.edu.iq \\ ${ }^{1}$ Department of Mathematics, \\ University of Reading, \\ Whiteknights, Reading, UK \\ ${ }^{2}$ Faculty of Computer Science \\ and Mathematics, University of \\ Kufa, Najaf, Iraq
}

\begin{abstract}
CMO functions multiplicative functions $f$ for which $\sum_{n=1}^{\infty} f(n)=0$. Such functions were first defined and studied by Kahane and Saïas [14]. We generalised these to Beurling prime systems with the aim to investigate the theory of the extended functions and we shall call them $C M O_{\mathcal{P}}$ functions. We give some properties and find examples of $C M O_{\mathcal{P}}$ functions. In particular, we explore how quickly the partial sum of these classes of functions tends to zero with different generalised prime systems. The findings of this paper may suggest that for all $C M O_{\mathcal{P}}$ functions $f$ over $\mathcal{N}$ with abscissa 1, we have

$$
\sum_{\substack{n \leq x \\ n \in \mathcal{N}}} f(n)=\Omega\left(\frac{1}{\sqrt{x}}\right)
$$
\end{abstract}

Keywords: Beurling's generalized primes, Multiplicative functions

Mathematics Subject Classification: 11N80, 11N56

\section{Introduction}

\subsection{Completely multiplicative function with sum zero}

A function $f: \mathbb{N} \longrightarrow \mathbb{C}$ is called a completely $C M O$ function if it satisfies the two following conditions:

$$
f \text { is a completely multiplicative function and } \sum_{n=1}^{\infty} f(n)=0 \text {. }
$$

Such functions were first introduced by Kahane and Saïas [14]. One motivation for them is to gain further insight into the zeros of Dirichlet series with completely multiplicative coefficients. Namely, the Generalised Riemann Hypothesis as discussed below. They also gave some properties and examples of such functions. For instance, they discussed various examples of CMO functions including $f(n)=\frac{\lambda(n)}{n}$, where $\lambda(n)$ is the Liouville function and $f(n)=\frac{\chi(n)}{n^{\alpha}}$, where $\chi$ is a non-principal Dirichlet character and $\alpha$ is a zero of $L_{\chi}$ with $\Re \alpha>0$.

This study led them to consider the question of how quickly $\sum_{n \leq x} f(n)$ can tend to zero. They suggested that it is always $\Omega\left(\frac{1}{\sqrt{x}}\right)$ and the Generalised Riemann HypothesisRiemann Hypothesis (GRH-RH) would follow if their statement is true. This suggestion author(s) and the source, provide a link to the Creative Commons licence, and indicate if changes were made. The images or other third party material in this article are included in the article's Creative Commons licence, unless indicated otherwise in a credit line to the material. If material is not included in the article's Creative Commons licence and your intended use is not permitted by statutory regulation or exceeds the permitted use, you will need to obtain permission directly from the copyright holder. To view a copy of this licence, visit http://creativecommons.org/licenses/by/4.0/. 
is incredibly difficult to prove, but it might be easier to disprove; i.e., to find examples such that

$$
\sum_{n \leq x} f(n)=O\left(\frac{1}{x^{c}}\right) \text { for some } c>\frac{1}{2}
$$

To date no such counter examples have been found. One approach to consider this counter example question is to consider examples of generalised $C M O$ functions on Beurling prime systems.

\subsection{Beurling generalised prime systems}

The concept of generalised primes and generalised integers was introduced by Beurling [3] in the 1930s and has been studied by many researchers since then (see for instance $[2,4,11,15,18])$. The structure of this system is defined to be a sequence of real positive numbers $\mathcal{P}=\left\{p_{1}, p_{2}, p_{3}, \ldots\right\}$ which need not be actual primes (or even integers) satisfying

$$
1<p_{1} \leq p_{2} \leq \cdots \leq p_{i} \leq \cdots
$$

and for which $p_{i} \longrightarrow \infty$ as $i \longrightarrow \infty$. With this sequence we can form a new increasing sequence

$$
1<n_{1} \leq n_{2} \leq \cdots \leq n_{i} \leq \cdots
$$

of real numbers which represent all possible products $\prod_{i=1}^{k} p_{i}^{a_{i}}$, where $k \in \mathbb{N}$ and $a_{1}, a_{2}, \ldots, a_{k} \in \mathbb{N}_{0}{ }^{1}$ These new elements are called generalised integers associated to $\mathcal{P}$ and denoted by $\mathcal{N}$; (i.e. $\mathcal{N}=\left\{n_{i}\right\}_{i \geq 1}$ ). Attached to these systems we have the usual counting functions $\pi_{\mathcal{P}}(x)$ and $N_{\mathcal{P}}(x)$ which are the sum over all the $g$-primes and $g$-integers not exceeding the positive real number $x$, counting multiplicities, respectively; that is

$$
\pi_{\mathcal{P}}(x)=\sum_{\substack{p \leq x \\ p \in \mathcal{P}}} 1 \text { and } N_{\mathcal{P}}(x)=\sum_{\substack{n \leq x \\ n \in \mathcal{N}}} 1 .
$$

These type of systems are discrete systems, where $\pi$ and $N$ are step functions with integer jumps. There is also a concept of continuous $g$-prime systems $[8,12]$, but they shall not concern us here. The generalised zeta function, the associated zeta function, is formally defined by

$$
\zeta_{\mathcal{P}}(s)=\prod_{p \in \mathcal{P}} \frac{1}{1-p^{-s}}=\sum_{n \in \mathcal{N}} \frac{1}{n^{s}} .
$$

We say that a $g$-prime system $\mathcal{P}$ has an abscissa of convergence $\sigma_{c}$ if and only if the sum of (1) converges for $\Re s>\sigma_{c}$ and diverges for $\Re s<\sigma_{c}$. The product is called the Euler product of the Beurling zeta function. The sum of (1) represents the generalised Dirichlet series which will be generated by multiplying out this product in the same way as the standard Euler product, defined in many books and papers.

In this paper, we outline some relevant ideas and results about $g$-primes and $g$-integers, in order to prove the main results in this paper, where we are interested 
in g-prime systems for which both $N_{\mathcal{P}}(x)$ and $\psi_{\mathcal{P}}(x)$ are simultaneously "well-behaved". These systems were investigated by Hilberdink [11] and have the following properties:

$$
N_{\mathcal{P}}(x)=\rho x+O\left(x^{\beta+\varepsilon}\right) \text { for some } \rho>0
$$

and

$$
\psi_{\mathcal{P}}(x)=x+O\left(x^{\alpha+\varepsilon}\right)
$$

hold for all $\varepsilon>0$, but for no $\varepsilon<0$ and $0 \leq \alpha, \beta<1$. For the usual primes, (2) holds with $\beta=0$ and if the RH is true, then (3) would hold for $\alpha=\frac{1}{2}$. Such systems exist as was shown by Zhang [17]. Indeed, $\mathcal{P}_{Z}$ (his system) satisfies these with $\alpha=\beta=\frac{1}{2}$. We also study such systems where either of (2) or (3) holds with $\alpha, \beta<\frac{1}{2}$.

\subsection{Completely multiplicative function on $\mathcal{N}$}

An arithmetical function with domain $\mathcal{N}$ is a function $f: \mathcal{N} \longrightarrow \mathbb{C}$ which is defined on the multi-set of Beurling integers $\mathcal{N}$.

Note that we are abusing the notion of function in case of multiplicities. This is done for clarity of notation. In much of our work, we are not interested in the arithmetic function $f: \mathcal{N} \longrightarrow \mathbb{C}$ itself, but in the partial sum of the function $f(n)$ up to and including $x$; i.e.

$$
\sum_{\substack{n \leq x \\ n \in \mathcal{N}}} f(n)
$$

which is a function because it counts all the possible elements of $\mathcal{N}$ up to $x$. A function $f: \mathcal{N} \longrightarrow \mathbb{C}$ is said to be multiplicative on $\mathcal{N}$ if $f(1)=1$ and $f(m n)=f(m) f(n)$ whenever $(m, n)=1$. Such an $f$ is said to be completely multiplicative $[5,16]$ if we also have $f(m n)=f(m) f(n)$ for all values of $m, n \in \mathcal{N}$, where $(m, n)$ is defined as the largest $g$ integer that divides both $m$ and $n$. We define the generalised Liouville function on $\mathcal{N}$, is an example of completely multiplicative function, to be $\lambda_{\mathcal{P}}(1)=1$ and $\lambda_{\mathcal{P}}(n)=(-1)^{a_{1}+\cdots+a_{k}}$ for $n=p_{1}^{a_{1}} \cdots p_{k}^{a_{k}} \in \mathcal{N}$, where $k \in \mathbb{N}$ and $a_{1}, \ldots, a_{k} \in \mathbb{N}_{0}$. As for classical multiplicative functions, if $f$ and $g$ are multiplicative functions and $f\left(p^{k}\right)=g\left(p^{k}\right)$ for all $g$-primes $p \in \mathcal{P}$ and $k \in \mathbb{N}_{0}$, then $f=g$.

We also define the generalised Chebyshev function over $\mathcal{N}$ with the sum extending over all $g$-prime numbers $p \in \mathcal{P}$ that are less than or equal to $x$ as follows:

$$
\psi_{\mathcal{P}}(x)=\sum_{\substack{p^{k} \leq x \\ p \in \mathcal{P} \\ k \in \mathbb{N}}} \log p .
$$

As in classical prime number theory, we introduce the $g$-prime counting function

$$
\Pi_{\mathcal{P}}(x)=\sum_{n=1}^{\infty} \frac{\pi_{\mathcal{P}}\left(x^{\frac{1}{n}}\right)}{n} .
$$

It is related to $\psi_{\mathcal{P}}(x)$ via

$$
\psi_{\mathcal{P}}(x)=\int_{p_{1}}^{x} \log t d \Pi_{\mathcal{P}}(t)
$$

We can also define the functions $l_{\mathcal{P}}$ and $m_{\mathcal{P}}$ which represent the following partial sums:

$$
l_{\mathcal{P}}(x):=\sum_{\substack{n \leq x \\ n \in \mathcal{N}}} \frac{\lambda_{\mathcal{P}}(n)}{n} \quad \text { and } \quad m_{\mathcal{P}}(x):=\sum_{\substack{n \leq x \\ n \in \mathcal{N}}} \frac{\mu_{\mathcal{P}}(n)}{n} .
$$




\subsection{Relationship between $\lambda_{\mathcal{P}}$ and $\mu_{\mathcal{P}}$}

In this section, we derive results which establish relationships between the $\lambda_{\mathcal{P}}$ and $\mu_{\mathcal{P}}$ functions as in the classical case. Of course, we shall always be aware that these are not necessarily functions if they are made from different $g$-primes. We first introduce two useful propositions and described as follows, which their proofs are standard.

Proposition 1 For every $n \in \mathcal{N}$, we have

$$
\lambda_{\mathcal{P}}(n)=\sum_{\substack{d^{2} \mid n \\ d \in \mathcal{N}}} \mu_{\mathcal{P}}\left(\frac{n}{d^{2}}\right) .
$$

Proposition 2 For every $n \in \mathcal{N}$, we have

$$
\mu_{\mathcal{P}}(n)=\sum_{\substack{d^{2} \mid n \\ d \in \mathcal{N}}} \lambda_{\mathcal{P}}\left(\frac{n}{d^{2}}\right) \mu_{\mathcal{P}}(d) .
$$

As consequences of propositions 1 and 2 we have

$$
\begin{aligned}
l_{\mathcal{P}}(x) & =\sum_{\substack{n \leq x \\
n \in \mathcal{N}}} \sum_{\substack{d^{2} \mid n \\
d \in \mathcal{N}}} \frac{\mu_{\mathcal{P}}\left(\frac{n}{d^{2}}\right)}{n}=\sum_{\substack{d^{2} \leq x \\
d \in \mathcal{N}}} \sum_{\substack{n \leq x \leq t . d^{2} \mid n \\
n \in \mathcal{N}}} \frac{\mu_{\mathcal{P}}\left(\frac{n}{d^{2}}\right)}{n} \\
& =\sum_{\substack{d^{2} \leq x \\
d \in \mathcal{N}}} \sum_{\substack{m \leq \frac{x}{J^{*}} \\
m \in \mathcal{N}}} \frac{\mu_{\mathcal{P}}(m)}{m d^{2}}=\sum_{\substack{d^{2} \leq x \\
d \in \mathcal{N}}} \frac{m_{\mathcal{P}}\left(\frac{x}{d^{2}}\right)}{d^{2}}
\end{aligned}
$$

and

$$
\begin{aligned}
m_{\mathcal{P}}(x) & =\sum_{\substack{n \leq x \\
n \in \mathcal{N}}} \sum_{\substack{d^{2} \mid n \\
d \in \mathcal{N}}} \frac{\lambda_{\mathcal{P}}\left(\frac{n}{d^{2}}\right)}{n} \mu_{\mathcal{P}}(d)=\sum_{\substack{d^{2} \leq x \\
d \in \mathcal{N}}} \sum_{\substack{n \leq x \leq t, d^{2} \mid n \\
n \in \mathcal{N}}} \frac{\lambda_{\mathcal{P}}\left(\frac{n}{d^{2}}\right)}{n} \mu_{\mathcal{P}}(d) \\
& =\sum_{\substack{d^{2} \leq x \\
d \in \mathcal{N}}} \sum_{\substack{m \leq \frac{x}{d} \\
m \in \mathcal{N}}} \frac{\lambda_{\mathcal{P}}(m)}{m d^{2}} \mu_{\mathcal{P}}(d)=\sum_{\substack{d^{2} \leq x \\
d \in \mathcal{N}}} \frac{l_{\mathcal{P}}\left(\frac{x}{d^{2}}\right)}{d^{2}} \mu_{\mathcal{P}}(d) .
\end{aligned}
$$

Lemma 3 Let $\mathcal{P}$ be a g-prime system for which $\sum_{n \in \mathcal{N}} \frac{1}{n^{2}}$ converges. Then $N_{\mathcal{P}}(x)=$ $o\left(x^{2}\right)$.

Proof Since $\sum_{n \in \mathcal{N}} \frac{1}{n^{2}}$ converges and put

$$
A(x):=\sum_{\substack{n \leq x \\ n \in \mathcal{N}}} \frac{1}{n^{2}}=C+o(1),
$$

then, by Abel summation,

$$
\begin{aligned}
N_{\mathcal{P}}(x)=\sum_{\substack{n \leq x \\
n \in \mathcal{N}}} 1 & =A(x) \cdot x^{2}-2 \int_{1}^{x} A(t) t d t \\
& =(C+o(1)) x^{2}-2 \int_{1}^{x}(C+o(1)) t d t=o\left(x^{2}\right) .
\end{aligned}
$$


We now establish a relationship between $l_{\mathcal{P}}(x)$ and $m_{\mathcal{P}}(x)$ in terms of these sum functions tending to zero with increasing terms.

Theorem 4 Let $\mathcal{P}$ be a g-prime system for which $\sum_{n \in \mathcal{N}} \frac{1}{n^{2}}$ converges. Then $l_{\mathcal{P}}(x)=o(1)$ if and only if $m_{\mathcal{P}}(x)=o(1)$.

Proof Suppose $m_{\mathcal{P}}(x)=o(1)$. Let $\varepsilon>0$. Then $\left|m_{\mathcal{P}}(x)\right|<\varepsilon$ for $x \geq x_{0}$, some $x_{0}$. Thus $\left|m_{\mathcal{P}}\left(\frac{x}{d^{2}}\right)\right|<\varepsilon$ if $\frac{x}{d^{2}} \geq x_{0}$. Hence (4) gives

$$
\begin{aligned}
\left|l_{\mathcal{P}}(x)\right|=\sum_{\substack{d^{2} \leq x \\
d \in \mathcal{N}}} \frac{\left|m_{\mathcal{P}}\left(\frac{x}{d^{2}}\right)\right|}{d^{2}} & \leq \varepsilon \sum_{\substack{d^{2} \leq \frac{x}{d_{0}} \\
d \in \mathcal{N}}} \frac{1}{d^{2}}+\sum_{\substack{\frac{x}{x_{0}}<d^{2} \leq x \\
d \in \mathcal{N}}} \frac{\left|m_{\mathcal{P}}\left(\frac{x}{d^{2}}\right)\right|}{d^{2}} \\
& \leq \varepsilon \sum_{\substack{d^{2} \leq \leq x \\
d \in \mathcal{N}}} \frac{1}{d^{2}}+A \frac{x_{0}}{x} \sum_{\substack{\frac{x}{x_{0}}<d^{2} \leq x \\
d \in \mathcal{N}}} 1 \quad\left(\text { since }\left|m_{\mathcal{P}}\left(\frac{x}{d^{2}}\right)\right| \leq A\right) \\
& \leq \varepsilon \zeta_{\mathcal{P}}(2)+A x_{0} \frac{N_{\mathcal{P}}(\sqrt{x})}{x} .
\end{aligned}
$$

Letting $x \longrightarrow \infty$ and using $N_{\mathcal{P}}(\sqrt{x})=o(x)$ (by Lemma 3), we find

$$
\limsup _{x \longrightarrow \infty}\left|l_{\mathcal{P}}(x)\right| \leq \varepsilon \zeta \mathcal{P}(2) .
$$

This is true for all $\varepsilon>0$. Since $\varepsilon$ is arbitrary, then $l_{\mathcal{P}}(x) \rightarrow 0$.

Now suppose $l_{\mathcal{P}}(x)=o(1)$. Let $\varepsilon>0$. Then $\left|l_{\mathcal{P}}(x)\right|<\varepsilon$ for $x \geq x_{0}$, some $x_{0}$. Thus $\left|l_{\mathcal{P}}\left(\frac{x}{d^{2}}\right)\right|<\varepsilon$ if $\frac{x}{d^{2}} \geq x_{0}$. Hence (5) gives

$$
\begin{aligned}
& \left|m_{\mathcal{P}}(x)\right|=\sum_{\substack{d^{2} \leq x \\
d \in \mathcal{N}}} \frac{\left|l_{\mathcal{P}}\left(\frac{x}{d^{2}}\right) \cdot \mu_{\mathcal{P}}(d)\right|}{d^{2}} \leq \varepsilon \sum_{\substack{d^{2} \leq \frac{x}{x^{2}} \\
d \in \mathcal{N}}} \frac{1}{d^{2}} \\
& +\sum_{\substack{x \\
\frac{x}{x_{0}}<d^{2} \leq x \\
d \in \mathcal{N}}} \frac{\left|l_{\mathcal{P}}\left(\frac{x}{d^{2}}\right)\right|}{d^{2}} \quad\left(\text { since }\left|\mu_{\mathcal{P}}(d)\right| \leq 1\right) \\
& \left.\leq \varepsilon \sum_{\substack{d^{2} \leq \frac{x}{x_{0}} \\
d \in \mathcal{N}}} \frac{1}{d^{2}}+A \frac{x_{0}}{x} \sum_{\substack{x \\
\frac{x}{x_{0}}<d^{2} \leq x \\
d \in \mathcal{N}}} 1 \quad \text { (since }\left|l_{\mathcal{P}}\left(\frac{x}{d^{2}}\right)\right| \leq A\right) \\
& \leq \varepsilon \zeta_{\mathcal{P}}(2)+A x_{0} \frac{N_{\mathcal{P}}(\sqrt{x})}{x} \text {. }
\end{aligned}
$$

Letting $x \longrightarrow \infty$ and using $N_{\mathcal{P}}(\sqrt{x})=o(x)$ (by Lemma 3), we find

$$
\limsup _{x \longrightarrow \infty}\left|m_{\mathcal{P}}(x)\right| \leq \varepsilon \zeta_{\mathcal{P}}(2) .
$$

This is true for all $\varepsilon>0$. Since $\varepsilon$ is arbitrary, then $m_{\mathcal{P}}(x) \rightarrow 0$.

Remark 5 In particular, if the abscissa of the $g$-prime system $\mathcal{P}$ is 1 , then $m_{\mathcal{P}}(x)$ and $l_{\mathcal{P}}(x)$ tend to zero together.

The following theorem establishes a useful correspondence between

$$
M_{\mathcal{P}}(x)=\sum_{\substack{n \leq x \\ n \in \mathcal{N}}} \mu_{\mathcal{P}}(n) \quad \text { and } \quad L_{\mathcal{P}}(x)=\sum_{\substack{n \leq x \\ n \in \mathcal{N}}} \lambda_{\mathcal{P}}(n) .
$$


Theorem 6 Let $\mathcal{P}$ be a g-prime system with abscissa 1 and let $\frac{1}{2}<a \leq 1$. Then $L_{\mathcal{P}}(x)=$ $O\left(x^{a}\right)$ if and only if $M_{\mathcal{P}}(x)=O\left(x^{a}\right)$.

Proof Assume $M_{\mathcal{P}}(x)=O\left(x^{a}\right)$. Then, in the same way that we obtained (4), we have

$$
L_{\mathcal{P}}(x)=\sum_{\substack{d^{2} \leq x \\ d \in \mathcal{N}}} M_{\mathcal{P}}\left(\frac{x}{d^{2}}\right) \ll \sum_{\substack{d \leq \sqrt{x} \\ d \in \mathcal{N}}} \frac{x^{a}}{d^{2 a}} \leq x^{a} \zeta_{\mathcal{P}}(2 a)
$$

since $2 a>1$, so $\zeta_{\mathcal{P}}(2 a)$ exists.

For the converse, assume $L_{\mathcal{P}}(x)=O\left(x^{a}\right)$. Since $\left|\mu_{\mathcal{P}}(d)\right| \leq 1$, in the same way that we obtained (5), we have

$$
M_{\mathcal{P}}(x)=\sum_{\substack{d^{2} \leq x \\ d \in \mathcal{N}}} L_{\mathcal{P}}\left(\frac{x}{d^{2}}\right) \mu_{\mathcal{P}}(d) \ll x^{a} \sum_{\substack{d \leq \sqrt{x} \\ d \in \mathcal{N}}} \frac{1}{d^{2 a}}=x^{a} \zeta_{\mathcal{P}}(2 a) .
$$

\section{Completely multiplicative function with sum zero on $\mathcal{N}$}

Let $\mathcal{P}$ be a $g$-prime system. We say that $f: \mathcal{N} \longrightarrow \mathbb{C}$ is a $\mathrm{CMO}_{\mathcal{P}}$ function if it satisfies the following conditions:

$$
\text { (i) } f \text { is a completely multiplicative function } \quad \text { (ii) } \sum_{n \in \mathcal{N}} f(n)=0 \text {. }
$$

This is a generalisation of a $C M O$ function. We investigate some properties of $C M O_{\mathcal{P}}$ functions. The same questions that were asked by Kahane and Saias [14] about $C M O$ functions can be discussed for $C M O_{\mathcal{P}}$ functions. For example, how quickly $\sum_{n \leq x} f(n)$ can tend to zero. In particular, we would like to investigate how quickly the partial sum of $\lambda_{\mathcal{P}}(n)$ over $n$ up to and including $x$ tends to zero with different type of systems where $\lambda_{\mathcal{P}}(n)$ is Liouville's function over $\mathcal{N}$. Specially, we discuss $O$-Results of $\sum_{n \leq x} \frac{\lambda_{\mathcal{P}}(n)}{n}$ over $\mathcal{N}$ with a system which satisfies

$$
N_{\mathcal{P}}(x)=\rho x+O\left(x^{\beta+\varepsilon}\right) \quad(\text { for some } \rho>0) \quad \text { and } \quad \psi_{\mathcal{P}}(x)=x+O\left(x^{\alpha+\varepsilon}\right)
$$

for all $\varepsilon>0$, but for no $\varepsilon<0$ and $0 \leq \alpha, \beta<1$. As special case we treat Zhang's system (see Theorem 1 of [17]) with error term $O\left(x^{\frac{1}{2}} e^{(c \log x)^{\frac{2}{3}}}\right)$ for the counting function $N_{\mathcal{P}}(x)$ and $O\left(x^{\frac{1}{2}}\right)$ for $\psi_{\mathcal{P}}(x)$. We show that $\sum_{n \leq x} \frac{\lambda_{\mathcal{P}}(n)}{n}$ for the system which satisfies (6) is $O\left(\frac{1}{x^{1-\Theta-\varepsilon}}\right)$, where $\Theta$ is the maximum value between $\alpha$ and $\beta$, whereas Zhang's system gives

$$
L_{\mathcal{P}}(x)=O\left(x^{\frac{1}{2}} e^{(c \log x)^{\frac{2}{3}}}\right)
$$

where $L_{\mathcal{P}}(x)$ is the partial sum of the Liouville function on $\mathcal{N}$ as defined previously. This can be compared to the conditional result of Balazard and de Roton [1] concerning the Möbius function of the standard integers. They showed that assuming RH,

$$
M(x)=O\left(x^{\frac{1}{2}} e^{(\log x)^{\frac{1}{2}}(\log \log x)^{\frac{5}{2}+\varepsilon}}\right) \text { for all } \varepsilon>0,
$$


where $M(x)$ is the partial sum of the Möbius function. Following the above result, Theorem 6 can be used to show that

$$
L(x)=O\left(x^{\frac{1}{2}} e^{c(\log x)^{\frac{1}{2}}(\log \log x)^{\frac{5}{2}+\varepsilon}}\right) \text { for all } \varepsilon>0 .
$$

We notice that the right hand side of (7) can be automatically improved if one would be able to improve the error term in Zhang's system.

We also explore $\Omega$ Results for the behaviour of $\sum_{n \leq x} \frac{\lambda_{\mathcal{P}}(n)}{n}$ for a system $\mathcal{P}$ which satisfies either the assumption

$$
N_{\mathcal{P}}(x)=\rho x+O\left(x^{\beta}\right) \text { for some } \rho>0 \text { or } \quad \psi_{\mathcal{P}}(x)=x+O\left(x^{\alpha}\right),
$$

for some $\alpha, \beta<\frac{1}{2}$. The aim is to find a completely multiplicative function $f$ over $\mathcal{N}$ with abscissa 1 such that

$$
\sum_{\substack{n \leq x \\ n \in \mathcal{N}}} f(n)=O\left(\frac{1}{x^{c}}\right) \text { for some } c>\frac{1}{2}
$$

\section{Some properties of $\mathrm{CMO}_{\mathcal{P}}$ functions}

In this section, we derive some preliminary properties of $C M O_{\mathcal{P}}$ functions.

Proposition 7 Let $f$ be a completely multiplicative function over $\mathcal{N}$. Then $\sum_{n \in \mathcal{N}}|f(n)|$ converges if and only if $\sum_{p \in \mathcal{P}}|f(p)|$ converges and $\sup _{p \in \mathcal{P}}|f(p)|<1$ for all $p \in \mathcal{P}$.

Proof It straightforward that the series $\sum_{p \in \mathcal{P}}|f(p)|$ converges and $\sup _{p \in \mathcal{P}}|f(p)|<1$ for all $p \in \mathcal{P}$ if $\sum_{n \in \mathcal{N}}|f(n)|$ converges.

Now suppose $\sum_{p \in \mathcal{P}}|f(p)|$ converges and $|f(p)|<1$ for all $p \in \mathcal{P}$. We want to prove $\sum_{n \in \mathcal{N}}|f(n)|$ converges.

From above, $c=\sup _{p \in \mathcal{P}}|f(p)|<1$. Therefore $\prod_{p \in \mathcal{P}} \frac{1}{1-|f(p)|}$ converges since

$$
1 \leq \prod_{p \in \mathcal{P}} \frac{1}{1-|f(p)|}=\prod_{p \in \mathcal{P}}\left(1+\frac{|f(p)|}{1-|f(p)|}\right) \leq \prod_{p \in \mathcal{P}}\left(1+\frac{1}{1-c}|f(p)|\right) .
$$

Now

$$
\begin{aligned}
\prod_{p \leq x} \frac{1}{1-|f(p)|} & =\sum_{\substack{n \in \mathcal{N} \\
p \mid n \& p \leq x}}|f(n)| \text { converges } \\
& \geq \sum_{n \leq x}|f(n)| .
\end{aligned}
$$

Hence $\sum_{n \in \mathcal{N}}|f(n)|$ converges.

Proposition 8 Letf be a $C M O_{\mathcal{P}}$ function. Then $\sum_{n \in \mathcal{N}}|f(n)|$ diverges. Indeed $\sum_{p \in \mathcal{P}}|f(p)|$ diverges.

Proof Let us assume the contrary, so that

$$
\sum_{n \in \mathcal{N}}|f(n)| \text { converges. }
$$

Then, by completely multiplicativity,

$$
\sum_{n \in \mathcal{N}}|f(n)|=\prod_{p \in \mathcal{P}} \frac{1}{1-|f(p)|} \quad \text { and } \quad \sum_{n \in \mathcal{N}} f(n)=\prod_{p \in \mathcal{P}} \frac{1}{1-f(p)} \neq 0 .
$$


This contradiction implies

$$
\sum_{n \in \mathcal{N}}|f(n)| \text { diverges. }
$$

Furthermore, Proposition 7 gives $\sum_{p \in \mathcal{P}}|f(p)|$ diverges, as required.

\subsection{Partial sums of $C M O_{\mathcal{P}}$ functions}

We know that the partial sum of an $C M O_{\mathcal{P}}$ function not exceeding $x$ tends to zero when $x$ tends to infinity. A question raised by Kahane and Saïas [14] regarding $C M O$ functions is: can one show, given $g(x)$, that there exists a $C M O$ function $f$ with

$$
\sum_{n \leq x} f(n)=\Omega(g(x)) ?
$$

We are not considering this question, but we are interested in a related question which is: how small can we make $g(x)$, so that the above is true for all $C M O_{\mathcal{P}}$ functions $f$ ? To answer this we need some assumptions on $g$-prime systems.

Proposition 9 Let $\mathcal{P}$ be a g-prime system such that all g-primes are distinct for which $\sum_{p \in \mathcal{P}} \frac{1}{p \log p}$ converges. Iff is a $C M O_{\mathcal{P}}$ function, then

$$
\sum_{\substack{n \leq x \\ n \in \mathcal{N}}} f(n)=\Omega\left(\frac{1}{x \log x}\right) .
$$

Proof Let us assume that the statement is false, so that

$$
\sum_{\substack{n \leq x \\ n \in \mathcal{N}}} f(n)=O\left(\frac{1}{x \log x}\right) .
$$

We know that for every $p \in \mathcal{P}$, we have

$$
f(p)=\sum_{m \leq p} f(m)-\sum_{m<p} f(m)=O\left(\frac{1}{p \log p}\right)
$$

Now it follows that $\sum_{p \in \mathcal{P}}|f(p)|$ converges and $|f(p)|<1$ for all $p \in \mathcal{P}$ by the assumptions. Hence, by Proposition 7, we have $\sum_{n \in \mathcal{N}}|f(n)|$ converges. However by Proposition 8 we have a contradiction, and so it follows that

$$
\sum_{\substack{n \leq x \\ n \in \mathcal{N}}} f(n)=\Omega\left(\frac{1}{x \log x}\right) .
$$

Remark $10 \quad$ (i) Similarly, we can get different results by having different assumptions on the $g$-prime systems such that all g-primes are distinct. ${ }^{2}$ For Example, if $f$ is a

${ }^{2}$ If all g-primes are not distinct, it can be more complicated. For instance, if $\mathcal{P}$ is $\{2,2,3,3, \ldots\}$ (every prime appears twice), then (8) does not work. 
$C M O_{\mathcal{P}}$ function and $\mathcal{P}$ is a $g$-prime system with such that all g-primes are distinct for which $\sum_{p \in \mathcal{P}} \frac{1}{p(\log \log p)^{2}}$ converges, then

$$
\sum_{\substack{n \leq x \\ n \in \mathcal{N}}} f(n)=\Omega\left(\frac{1}{x(\log \log x)^{2}}\right) .
$$

(ii) Kahane and Saïas [14] have shown that if $f$ is a CMO function, then

$$
\sum_{n \leq x} f(n)=\Omega\left(\frac{1}{x}\right)
$$

Now if we assume $\sum_{p \in \mathcal{P}} \frac{1}{p}$ converges, then the sum on the left of (9) is $\Omega\left(\frac{1}{x}\right)$; although the latter condition appears to be a bit unnatural in a number-theoretic context.

\subsection{Closeness relation between two completely multiplicative functions which are defined} over $\mathcal{N}$

Let $\mathcal{C} \mathcal{M}_{\mathcal{P}}:=\{f: \mathcal{N} \longrightarrow \mathbb{C}$ completely multiplicative $\}$, and let us define an (extended) metric on $\mathcal{C M}_{\mathcal{P}}$ to be the distance function

$$
D(f, g):=\sum_{p \in \mathcal{P}}|g(p)-f(p)| \text {. }
$$

Then $\mathcal{C} \mathcal{M}_{\mathcal{P}}$ is an extended metric space since $D(f, g)$ can attain the value $\infty$. We aim to generalise Theorem 3 of Kahane and Saïas [14] over Beurling prime systems.

The following theorem shows that if $f$ is a $C M O_{\mathcal{P}}$ function and $g$ is a nearby completely multiplicative function on $\mathcal{N}$, then $g$ is also a $C M O_{\mathcal{P}}$ function. In other words, under extra conditions on the values of $g$-primes for two completely multiplicative functions if one is a $C M O_{\mathcal{P}}$, then so is the other.

Theorem 11 Let $\mathcal{P}$ be a g-prime system with abscissa $1, f$ a $C M O_{\mathcal{P}}$ function and $g$ a completely multiplicative function on $\mathcal{P}$ such that

$$
|g(p)|<1 \text { for all } p \in \mathcal{P}
$$

and

$$
D(f, g)<\infty
$$

Then $g$ is a $\mathrm{CMO}_{\mathcal{P}}$ function.

Proof Let $F(s):=\sum_{n \in \mathcal{N}} \frac{f(n)}{n^{s}}$ and $G(s):=\sum_{n \in \mathcal{N}} \frac{g(n)}{n^{s}}$. Then the series for $F(s)$ is absolutely convergent for $\Re s>1$ and it is convergent for $\Re s>0$ and $s=0$ since $\sum_{n \in \mathcal{N}} f(n)=0$. Assumption (10) and that $g$ is completely multiplicative function imply $|g(n)| \leq 1$. Thus the series for $G(s)$ also converges for $\Re s>1$ since $g$ is bounded and the abscissa of $\mathcal{P}$ is 1 . Therefore $F(s)$ and $G(s)$ can be written as follows:

$$
F(s)=\prod_{p \in \mathcal{P}} \frac{1}{1-\frac{f(p)}{p^{s}}} \quad \text { and } \quad G(s)=\prod_{p \in \mathcal{P}} \frac{1}{1-\frac{g(p)}{p^{s}}} \quad \Re s>1 .
$$


Now

$$
H(s):=\prod_{p \in \mathcal{P}}\left(\frac{1-\frac{f(p)}{p^{s}}}{1-\frac{g(p)}{p^{s}}}\right)=\prod_{p}\left(1+\frac{\frac{g(p)-f(p)}{p^{s}}}{1-\frac{g(p)}{p^{s}}}\right)
$$

converges absolutely for $\Re s \geq 0$ if and only if

$$
\sum_{p \in \mathcal{P}} \frac{\left|\frac{g(p)-f(p)}{p^{s}}\right|}{\left|1-\frac{g(p)}{p^{s}}\right|}
$$

converges for $\Re s \geq 0$. But

$$
\frac{\left|\frac{g(p)-f(p)}{p^{s}}\right|}{\left|1-\frac{g(p)}{p^{s}}\right|} \leq 2 \frac{|g(p)-f(p)|}{p^{\Re s}}
$$

since $\left|1-\frac{g(p)}{p^{s}}\right| \geq \frac{1}{2}$ for $p$ sufficiently large and $\Re s>0$. Thus $H(s)$ converges absolutely to a holomorphic function for $\Re s>0$. However, $H(s)=(G / F)(s)$ for $\Re s>1$ then $G(s)=F(s) H(s)$, where the series for $F(s)$ converges for $\Re s>0$ and $s=0$ since $f$ is a $C M O_{\mathcal{P}}$ function, and $H(s)$ converges absolutely for $\Re s \geq 0$. Therefore $G(s)$ converges for $\Re s>0$ and $s=0$. Thus we have $G(0)=F(0) H(0)=0$. Hence $g$ is a $C M O_{\mathcal{P}}$ function.

The proof of Theorem 11 implies the following result.

Corollary 12 Let $\mathcal{P}$ be a g-prime system with abscissa $1, f$ and $g$ both be completely multiplicative functions on $\mathcal{P}$ such that $D(f, g)$ is finite and satisfying

$$
|f(p)|,|g(p)|<1 \text { for all } p \in \mathcal{P} .
$$

Then the following two assertions are equivalent:

$$
\sum_{n \in \mathcal{N}} f(n)=0 \text { and } \sum_{n \in \mathcal{N}} g(n)=0
$$

\section{The function $\frac{\lambda_{\mathcal{P}}(n)}{n}$ over different systems $\mathcal{P}$}

In this section, we provide some examples of $C M O_{\mathcal{P}}$ functions. In particular, we introduce some examples of the function $\frac{\lambda_{\mathcal{P}}(n)}{n}$ with different $g$-prime systems where $\lambda_{\mathcal{P}}(n)$ is the Liouville function over the $g$-prime system $\mathcal{P}$. We emphasize that we are only interested in those systems for which the abscissa of convergence of the Dirichlet series for $\zeta \mathcal{P}$ is 1 .

Example 13 As shown in $[6,7,9,10]$, if $\mathcal{P}$ satisfies one of the following assumptions:

$$
\psi_{\mathcal{P}}(x)=\int_{1}^{x} \frac{d\left(\psi_{\mathcal{P}}(t)\right)}{t}=\log x+c+o(1) \text { for some constant } c
$$

or

$$
M_{\mathcal{P}}(x)=o(x) \quad \text { and } \quad N_{\mathcal{P}}(x)-\rho x=O\left(\frac{x}{\log ^{\gamma} x}\right) \text { holds for some } \rho>0 \text { and } \gamma>1 \text {, }
$$

or

$$
M_{\mathcal{P}}(x)=o(x) \text { and } \quad \int_{1}^{\infty}\left|\frac{N_{\mathcal{P}}(x)-a x}{x^{2}}\right| d x<\infty \quad \text { for some } a>0,
$$


or

$$
N_{\mathcal{P}}(x) \sim \rho x \quad \text { and } \quad \log \zeta_{\mathcal{P}}(s)-\log \left(\frac{1}{s-1}\right) \quad \text { has a continuous extension to } \Re s=1 \text {, }
$$

or

$$
\int_{2}^{\infty}\left|\Pi_{\mathcal{P}}(x)-\frac{x}{\log x}\right| \frac{d x}{x^{2}}<\infty, \quad \text { where } \quad \Pi_{\mathcal{P}}(x)=\sum_{k=1}^{\infty} \frac{1}{k} \pi_{\mathcal{P}}\left(x^{\frac{1}{k}}\right)
$$

then $\sum_{n \in \mathcal{N}} \frac{\mu_{\mathcal{P}}(n)}{n}=0$. Therefore, by using Theorem $4, \sum_{n \in \mathcal{N}} \frac{\lambda_{\mathcal{P}}(n)}{n}=0$. Hence $\frac{\lambda_{\mathcal{P}}(n)}{n}$ is a $C M O_{\mathcal{P}}$ function since it is completely multiplicative with sum zero.

In fact, we do not even need $\pi_{\mathcal{P}}(x) \sim \frac{x}{\log x}$ for $\sum_{n \in \mathcal{N}} \frac{\lambda_{\mathcal{P}}(n)}{n}=0$ to be true since it is shown in [7] that the following condition

$$
\int_{2}^{\infty}\left|\Pi_{\mathcal{P}}(x)-\frac{x}{\log x}\left(1+\sum_{j=0}^{k} b_{j} \cos \left(t_{j} \log x+y_{j}\right)\right)\right| \frac{d x}{x^{2}}<\infty
$$

with distinct $t_{j}>0$ and $\left(1+t_{j}^{2}\right)^{1 / 2}\left|b_{j} \cos \left(y_{j}+\arctan t_{j}\right)\right|<2, j=1, \ldots, k$, is also sufficient to have $\sum_{n \in \mathcal{N}} \frac{\mu_{\mathcal{P}}(n)}{n}=0$.

From Example 13, we see that $\sum_{n \in \mathcal{N}} \frac{\lambda_{\mathcal{P}}(n)}{n}=0$, but how quickly does it converge?

\subsection{O-results for Euler's example over $\mathcal{N}$}

In this section, we shall be interested in the behaviour of $\sum_{n \leq x} \frac{\lambda_{\mathcal{P}}(n)}{n}$ over a $g$-prime system $\mathcal{P}$ which satisfies

$$
N_{\mathcal{P}}(x)=\rho x+O\left(x^{\beta+\varepsilon}\right) \quad(\text { for some } \rho>0)
$$

and

$$
\psi_{\mathcal{P}}(x)=x+O\left(x^{\alpha+\varepsilon}\right)
$$

as $x \longrightarrow \infty$ for all $\varepsilon>0$, but for no $\varepsilon<0$ and $0 \leq \alpha, \beta<1$.

Theorem 14 Given a g-prime system $\mathcal{P}$ satisfying (19) and (20) for some $\beta, \alpha<1$, and let $\lambda_{\mathcal{P}}$ as defined before, we have

$$
\sum_{\substack{n \leq x \\ n \in \mathcal{N}}} \frac{\lambda_{\mathcal{P}}(n)}{n}=O\left(\frac{1}{x^{1-\Theta-\varepsilon}}\right) \text { for all } \varepsilon>0
$$

where $\Theta=\max \{\alpha, \beta\}$.

Proof We let $\mathcal{P}$ denote a g-prime system satisfying (19) and (20). Then, for all $\Re s>1$, we have

$$
Z_{\mathcal{P}}(s)=\sum_{n \in \mathcal{N}} \frac{\lambda_{\mathcal{P}}(n)}{n^{s}}=\frac{\zeta_{\mathcal{P}}(2 s)}{\zeta_{\mathcal{P}}(s)}
$$


We need a bound for $Z_{\mathcal{P}}(s)$ on vertical line $s=\sigma+i t$ with $|t|$ large and $\sigma>\Theta$. To find such bound of $Z_{\mathcal{P}}(s)$, we start with

$$
\left|\zeta_{\mathcal{P}}(2 s)\right|=\left|\sum_{n \in \mathcal{N}} \frac{1}{n^{2 \sigma+2 i t}}\right| \leq \sum_{n \in \mathcal{N}} \frac{1}{n^{2 \sigma}}=O(1)
$$

for $\sigma>\frac{1}{2}$.

From the proof of Theorem 2.3 of [13], for $\sigma>\Theta$, we have

$$
\log \left|\zeta_{\mathcal{P}}(\sigma+i t)\right|=O\left((\log |t|)^{\frac{1-\sigma}{1-\Theta}+\varepsilon}\right) .
$$

In particular, for $\sigma>\Theta$,

$$
\frac{1}{\left|\zeta_{\mathcal{P}}(\sigma+i t)\right|}=O\left(|t|^{\varepsilon}\right) \text { for all } \varepsilon>0 .
$$

Hence, for $\sigma>\Theta$,

$$
\left|Z_{\mathcal{P}}(s)\right|=\left|\frac{\zeta_{\mathcal{P}}(2 \sigma+2 i t)}{\zeta_{\mathcal{P}}(\sigma+i t)}\right|=O\left(|t|^{\varepsilon}\right) \quad \text { for all } \varepsilon>0 \text { and for }|t| \geq 1
$$

Using the inverse Mellin transform we have for $x>0, x \notin \mathcal{N}$

$$
L_{\mathcal{P}}(x)=\frac{1}{2 \pi i} \int_{c-i \infty}^{c+i \infty} \frac{Z_{\mathcal{P}}(s)}{s} x^{s} d s \quad(c>1) .
$$

Now split the range into $(c-i \infty, c-i T],[c-i T, c+i T]$ and $[c+i T, c+i \infty)$, where $T>0$ is a suitable function of $x$ which will be chosen later, we obtain

$$
L_{\mathcal{P}}(x)=\frac{1}{2 \pi i} \int_{c-i T}^{c+i T} \frac{Z_{\mathcal{P}}(s)}{s} x^{s} d s+\frac{1}{2 \pi i}\left(\int_{c+i T}^{c+i \infty}+\int_{c-i \infty}^{c-i T}\right) \frac{Z_{\mathcal{P}}(s)}{s} x^{s} d s .
$$

Denote the left integral by $I_{1}$. We note that assumption (19) implies $\zeta_{\mathcal{P}}(s)$ has an analytic continuation to $\{s \in \mathbb{C}: \Re s>\beta\}$ except for a simple pole at $s=1$. This implies $\left(U_{\mathcal{P}}(s)=\right) \frac{1}{\zeta_{\mathcal{P}}(s)}$ is holomorphic for $\Re^{\prime} s>\beta$. However, assumption $(20)$ implies that $\zeta_{\mathcal{P}}(s)$ is holomorphic for $\Re s>\alpha$ except for a simple pole at $s=1$ and $\zeta_{\mathcal{P}}(s) \neq 0$ in this region (see Theorem 2.1 of [13]). Thus (19) and (20) together show $\zeta_{\mathcal{P}}(s)$ is holomorphic and has no zeros for $\Re s>\Theta$ except for a simple pole at $s=1$. Hence $U_{\mathcal{P}}(s)$ has a simple zero at $s=1$ and an analytic continuation to $\{s \in \mathbb{C}: \Re s>\Theta\}$. Thus $\left(Z_{\mathcal{P}}(s)=\right) \frac{\zeta_{\mathcal{P}}(2 s)}{\zeta_{\mathcal{P}}(s)}$ has an analytic continuation to $\{s \in \mathbb{C}: \Re s>\Theta\}$ with a simple zero at $s=1$. Hence $Z_{\mathcal{P}}(s)$ is holomorphic for $\sigma>\Theta$ since $\zeta_{\mathcal{P}}(2 s)$ is holomorphic for $\sigma>\Theta$ because $\Theta \geq \frac{1}{2}$ (see [13]).

Now move the contour past the line $s=1$ to the line $\Re s=\sigma$ for any $\sigma>\Theta$ since $Z_{\mathcal{P}}(s)$ is holomorphic in this region. Hence

$$
I_{1}=\frac{1}{2 \pi i}\left(\int_{c-i T}^{\sigma-i T}+\int_{\sigma-i T}^{\sigma+i T}+\int_{\sigma+i T}^{c+i T}\right) \frac{Z_{\mathcal{P}}(s)}{s} x^{s} d s .
$$

These integrals will be estimated by using the bound $\left|Z_{\mathcal{P}}(s)\right|=O\left(t^{\varepsilon}\right)$ for all $\varepsilon>0$. The integral over the horizontal path $[\sigma+i T, c+i T]$ is 


$$
\left|\frac{1}{2 \pi i} \int_{\sigma+i T}^{c+i T} \frac{Z_{\mathcal{P}}(s)}{s} x^{s} d s\right| \leq \frac{x^{c}}{2 \pi T} \int_{\sigma}^{c}\left|Z_{\mathcal{P}}(y+i T)\right| d y=O\left(\frac{x^{c}}{T^{1-\varepsilon}}\right) \text { for all } \varepsilon>0,
$$

by (21). Similarly for the integral over $[c-i T, \sigma-i T]$. On the line $\Re s=\sigma$, we will have

$$
\left|\frac{1}{2 \pi i} \int_{\sigma-i T}^{\sigma+i T} \frac{Z_{\mathcal{P}}(s)}{s} x^{s} d s\right| \leq \frac{x^{\sigma}}{2 \pi} \int_{-T}^{T} \frac{\left|Z_{\mathcal{P}}(\sigma+i t)\right|}{|\sigma+i t|} d t=O\left(x^{\sigma} T^{\varepsilon}\right) \quad \text { for all } \varepsilon>0,
$$

by (21). Hence

$$
I_{1}=O\left(\frac{x^{c}}{T^{1-\varepsilon}}\right)+O\left(x^{\sigma} T^{\varepsilon}\right)
$$

Now the right integral of (22) will be estimated as follows:

$$
\left|I_{2}\right| \leq \sum_{n \in \mathcal{N}}\left|\lambda_{\mathcal{P}}(n)\right| \cdot\left|\frac{1}{2 \pi i}\left(\int_{c+i T}^{c+i \infty}+\int_{c-i \infty}^{c-i T}\right)\left(\frac{x}{n}\right)^{s} \frac{d s}{s}\right|=O\left(\frac{x^{c}}{T} \sum_{n \in \mathcal{N}} \frac{1}{n^{c}\left|\log \frac{x}{n}\right|}\right)
$$

The range is split into $\left(n \geq 2 x\right.$ and $\left.n \leq \frac{x}{2}\right)$ and $\left(\frac{x}{2}<n<2 x\right)$ in order to use the bound $\left|\log \frac{x}{n}\right| \geq \log 2$ for the first range. This gives

$$
I_{2}=O\left(\frac{x^{c}}{T} \sum_{\substack{n \geq 2 x \& n \leq \frac{x}{2} \\ n \in \mathcal{N}}} \frac{1}{n^{c}\left|\log \frac{x}{n}\right|}\right)+O\left(\frac{x^{c}}{T} \sum_{\substack{\frac{x}{2}<n<2 x \\ n \in \mathcal{N}}} \frac{1}{n^{c}\left|\log \frac{x}{n}\right|}\right) .
$$

Using $\left|\log \frac{x}{n}\right|=\left|\log \left(1+\frac{n-x}{x}\right)\right| \asymp \frac{|n-x|}{x}$ for the second range, we obtain

$$
\begin{aligned}
I_{2} & =O\left(\frac{x^{c}}{T} \sum_{\substack{n \geq 2 x \& n \leq \frac{x}{2} \\
n \in \mathcal{N}}} \frac{1}{n^{c}}\right)+O\left(\frac{x}{T} \sum_{\substack{\frac{x}{2}<n<2 x \\
n \in \mathcal{N}}} \frac{1}{|n-x|}\right) \\
& =O\left(\frac{x^{c}}{T} \cdot \zeta_{\mathcal{P}}(c)\right)+O\left(\frac{x}{T} \sum_{\substack{x \\
\frac{x}{2}<n<22 \\
n \in \mathcal{N}}} \frac{1}{|n-x|}\right)
\end{aligned}
$$

Therefore

$$
L_{\mathcal{P}}(x)=I_{1}+I_{2}=O\left(\frac{x^{c}}{T^{1-\varepsilon}}\right)+O\left(x^{\sigma} T^{\varepsilon}\right)+O\left(\frac{x^{c}}{T} \cdot \zeta_{\mathcal{P}}(c)\right)+O\left(\frac{x}{T} \sum_{\substack{x \\ \frac{x}{2}<n<2 x \\ n \in \mathcal{N}}} \frac{1}{|n-x|}\right)
$$

Taking $c=1+\frac{1}{\log x}$ and using $\zeta_{\mathcal{P}}(1+\delta)=O\left(\frac{1}{\delta}\right)$, gives

$$
L_{\mathcal{P}}(x)=O\left(\frac{x}{T^{1-\varepsilon}}\right)+O\left(x^{\sigma} T^{\varepsilon}\right)+O\left(\frac{x \log x}{T}\right)+O\left(\frac{x}{T} \sum_{\substack{x \\ \frac{x}{2}<n<2 x \\ n \in \mathcal{N}}} \frac{1}{|n-x|}\right)
$$

for $x \notin \mathcal{N}$ and for all $\varepsilon>0$. We need to bound the term on the right hand side, which is difficult for general $x$ when $n$ is an integer close to $x$, as then $|n-x|^{-1}$ could be very large. To take into account this eventuality we choose $x$ here such that $|n-x|<\frac{1}{x^{2}}$. This ensures that it stays away from these integer $n$; i.e.

$$
\left(x-\frac{1}{x^{2}}, x+\frac{1}{x^{2}}\right) \cap \mathcal{N}=\phi .
$$


Then, for $\operatorname{such} x$,

$$
\sum_{\substack{\frac{x}{2}<n<2 x \\ n \in \mathcal{N}}} \frac{1}{|n-x|} \leq x^{2} \cdot \sum_{\substack{x \\ \frac{x}{2}<n<2 x \\ n \in \mathcal{N}}} 1<x^{2} \cdot N(2 x)=O\left(x^{3}\right) .
$$

Hence, for such $x$,

$$
L_{\mathcal{P}}(x)=O\left(\frac{x}{T^{1-\varepsilon}}\right)+O\left(x^{\sigma} T^{\varepsilon}\right)+O\left(\frac{x \log x}{T}\right)+O\left(\frac{x^{4}}{T}\right) .
$$

Taking $T=x^{4}$, then

$$
\sum_{\substack{n \leq x \\ n \in \mathcal{N}}} \lambda_{\mathcal{P}}(n)=O\left(x^{\sigma+4 \varepsilon}\right) \quad \text { for all } \varepsilon>0
$$

Taking $\sigma=\Theta+\varepsilon$ for any $\varepsilon>0$, then

$$
\sum_{\substack{n \leq x \\ n \in \mathcal{N}}} \lambda_{\mathcal{P}}(n)=O\left(x^{\Theta+5 \varepsilon}\right)
$$

whenever $x$ is such that $\left(x-\frac{1}{x^{2}}, x+\frac{1}{x^{2}}\right) \cap \mathcal{N}=\phi$. Now we follow the method used in the proof of Theorem 2.1, originally given in (see [13, p. 30]). Exactly as in [13], one shows that

$$
\left(x_{1}-\frac{1}{x_{1}^{2}}, x_{1}+\frac{1}{x_{1}^{2}}\right) \cap \mathcal{N}=\phi \quad \text { and } \quad\left(x_{2}-\frac{1}{x_{2}^{2}}, x_{2}+\frac{1}{x_{2}^{2}}\right) \cap \mathcal{N}=\phi
$$

Then the result will follow since

$$
\sum_{\substack{n \leq x_{1} \\ n \in \mathcal{N}}} \lambda_{\mathcal{P}}(n)=O\left(x_{1}^{\Theta+5 \varepsilon}\right)=O\left(x^{\Theta+5 \varepsilon}\right) \text { for all } \varepsilon>0
$$

Hence

$$
\begin{aligned}
\sum_{\substack{n \leq x \\
n \in \mathcal{N}}} \lambda_{\mathcal{P}}(n)= & \sum_{\substack{n \leq x_{1} \\
n \in \mathcal{N}}} \lambda_{\mathcal{P}}(n) \\
& +\sum_{\substack{x_{1}<n \leq x \\
n \in \mathcal{N}}} \lambda_{\mathcal{P}}(n)=O\left(x^{\Theta+5 \varepsilon}\right)+O\left(x^{\beta+\varepsilon}\right)=O\left(x^{\Theta+5 \varepsilon}\right) \quad \text { for all } \varepsilon>0
\end{aligned}
$$

since

$$
\left|\sum_{\substack{x_{1}<n \leq x \\ n \in \mathcal{N}}} \lambda_{\mathcal{P}}(n)\right| \leq \sum_{\substack{x_{1}<n \leq x \\ n \in \mathcal{N}}} 1=N_{\mathcal{P}}(x)-N_{\mathcal{P}}\left(x_{1}\right) \leq N_{\mathcal{P}}(x)-N_{\mathcal{P}}(x-2)=O\left(x^{\beta+\varepsilon}\right) \quad \text { for all } \varepsilon>0,
$$

by (19). Thus

$$
L_{\mathcal{P}}(x)=\sum_{\substack{n \leq x \\ n \in \mathcal{N}}} \lambda_{\mathcal{P}}(n)=O\left(x^{\Theta+5 \varepsilon}\right) \text { for all } \varepsilon>0
$$

Now, by Abel Summation, this gives the result.

Remark $15 \quad$ (i) It was shown in Theorem 1 of [11] that $\Theta \geq \frac{1}{2}$. Thus, $1-\Theta \leq \frac{1}{2}$ and in Theorem 14, we can therefore only have an example with exponent $\leq \frac{1}{2}$ for such systems. 
(ii) If $\Theta=\frac{1}{2}$ in Theorem 14, then we have $l_{\mathcal{P}}(x)=O\left(\frac{e^{\varepsilon \log x}}{x^{\frac{1}{2}}}\right)$ for all $\varepsilon>0$. We can do slightly better than this bound if we take $\mathcal{P}=\mathcal{P}_{Z}$ (Zhang's system) which is a special case of a well-behaved system. In this case

$$
N_{\mathcal{P}}(x)=\rho x+O\left(x^{\frac{1}{2}} e^{c(\log x)^{\frac{2}{3}}}\right) \text { for some } \rho, c>0
$$

and

$$
\pi_{\mathcal{P}}(x)=\operatorname{li}(x)+O\left(x^{\frac{1}{2}}\right)
$$

hold. The existence of $\mathcal{P}_{Z}$ was shown by Zhang [17]. The proof of the following theorem is roughly identical with the previous theorem except that we need a strictly stronger bound on $Z_{\mathcal{P}}(s)$ than (21).

Theorem 16 For Zhang's system $\mathcal{P}_{Z}$, we have

$$
\sum_{\substack{n \leq x \\ n \in \mathcal{N}}} \frac{\lambda_{\mathcal{P}}(n)}{n}=O\left(\frac{e^{C(\log x)^{\frac{2}{3}}}}{x^{\frac{1}{2}}}\right) \text { for some constant } C .
$$

Remark 17 As in the proof of Theorem 14 the idea is to find a bound for $\sum_{n \leq x} \lambda_{\mathcal{P}}(n)$. As mentioned above, we need a stronger bound for $Z_{\mathcal{P}}(s)$. This can be found by using Theorem 7.11 of Zhang [10].

Proof of Theorem 16 We know from the proof of Theorem 7.11 of Zhang that there is a sequence of real numbers which represents a set of g-primes, (i.e. $\left.\mathcal{P}=\left\{p_{j}\right\}\right), j=1,2, \ldots$, such that

$$
\sum_{p_{j} \leq x} p_{j}^{-i t}-\int_{1}^{x} v^{-i t} f(v) d v \ll \sqrt{x}\left(1+\sqrt{\frac{\log (t+1)}{1+\log x}}\right)
$$

for $1 \leq x<\infty$ and $t \geq 0$, where

$$
f(v):=\frac{1-v^{-1}}{\log v} \text { for } v \geq 1
$$

In particular, when $t=0$,

$$
\sum_{p_{j} \leq x} 1-\int_{1}^{x} f(v) d v=O\left(x^{\frac{1}{2}}\right)
$$

and hence

$$
\pi_{\mathcal{P}}(x):=\sum_{p_{j} \leq x} 1=\operatorname{li}(x)+O\left(x^{\frac{1}{2}}\right) .
$$

Further Zhang showed that $\zeta_{\mathcal{P}}(s)$ can be written as

$$
\zeta_{\mathcal{P}}(s)=\frac{s}{s-1} \exp \left\{F_{2}(s)-F_{1}(s)\right\},
$$

where

$$
F_{1}(s)=\int_{1}^{\infty}\left(v^{-s}+\log \left(1-v^{-s}\right)\right) d \pi_{\mathcal{P}}(v)
$$


and

$$
F_{2}(s)=\int_{1}^{\infty} v^{-s}\left(d \pi_{\mathcal{P}}(v)-f(v) d v\right)
$$

Since

$$
\log \left(1-v^{-s}\right)=-v^{-s}+O\left(v^{-2 \sigma}\right)
$$

for $v>1$. Therefore

$$
F_{1}(s)=\int_{1}^{\infty}\left(v^{-s}+\log \left(1-v^{-s}\right)\right) d \pi_{\mathcal{P}}(v)=O\left(\int_{1}^{\infty} \frac{d \pi_{\mathcal{P}}(v)}{v^{2 \sigma}}\right) .
$$

The right integral converges uniformly for $\sigma>\frac{1}{2}$. Thus $F_{1}(s)$ is holomorphic for $\sigma>\frac{1}{2}$. By (26) and (27) that $F_{2}(s)$ is also holomorphic for $\sigma>\frac{1}{2}$. Hence $\zeta_{\mathcal{P}}(s)$ has an analytic continuation in the half plane $\sigma>\frac{1}{2}$ except for a simple pole at $s=1$ with residue $k=\exp \left\{F_{2}(1)-F_{1}(1)\right\}>0$. From (25), we have

$$
\begin{aligned}
\sum_{p_{j} \leq x} p_{j}^{-i t}-\int_{1}^{x} v^{-i t} f(v) d v & =\int_{1}^{x} v^{-i t} d \pi_{\mathcal{P}}(v)-\int_{1}^{x} v^{-i t} f(v) d v \\
& =\int_{1}^{x} v^{-i t}\left(d \pi_{\mathcal{P}}(v)-f(v) d v\right) \ll \sqrt{x}\left(1+\sqrt{\frac{\log (t+1)}{1+\log x}}\right) .
\end{aligned}
$$

Now, by Lemma 17.13 of [10], we have for $t \in \mathbb{R}$

$$
F_{2}(\sigma+i t) \ll \frac{1}{\sigma-\frac{1}{2}}+\sqrt{\frac{\log |t|}{\sigma-\frac{1}{2}}}
$$

uniformly for $\frac{1}{2}<\sigma \leq 2$. Furthermore, (28) imply that

$$
\begin{aligned}
F_{1}(\sigma+i t) \ll \int_{1}^{\infty} v^{-2 \sigma} d \pi_{\mathcal{P}}(v) & =2 \sigma \int_{1}^{\infty} v^{-2 \sigma-1} \pi_{\mathcal{P}}(v) d v \\
& \ll \int_{1}^{\infty} v^{-2 \sigma} d v \ll \frac{1}{\sigma-\frac{1}{2}}
\end{aligned}
$$

uniformly for $\frac{1}{2}<\sigma \leq 2$. Hence $\zeta_{\mathcal{P}}$ is holomorphic for $\frac{1}{2}<\sigma \leq 2$ except for a pole at $s=1$, and for these values satisfies uniformly

$$
F_{2}(s)-F_{1}(s) \ll\left(\frac{1}{\sigma-\frac{1}{2}}+\sqrt{\frac{\log |t|}{\sigma-\frac{1}{2}}}\right) .
$$

Then, for $\frac{1}{2}<\sigma \leq 2$ and $|t| \geq 1$, we have

$$
\log \left|\zeta_{\mathcal{P}}(\sigma+i t)\right| \ll \frac{1}{\sigma-\frac{1}{2}}+\sqrt{\frac{\log |t|}{\sigma-\frac{1}{2}}} .
$$

Hence

$$
\left|Z_{\mathcal{P}}(s)\right|=\left|\frac{\zeta_{\mathcal{P}}(2 \sigma+2 i t)}{\zeta_{\mathcal{P}}(\sigma+i t)}\right| \ll \exp \left\{C\left(\frac{1}{\sigma-\frac{1}{2}}\right)+C \sqrt{\frac{\log |t|}{\sigma-\frac{1}{2}}}\right\} .
$$

We know from Theorem 17.11 of Zhang in [10] that the function $\zeta_{\mathcal{P}}(s)$ has an analytic continuation to $\left\{s \in \mathbb{C}: \Re s>\frac{1}{2}\right\}$ except for a simple pole at $s=1$. Also, $\zeta_{\mathcal{P}}(s)$ has no zeros for $\sigma>\frac{1}{2}$ but $\frac{1}{\zeta \mathcal{P}(s)}$ has a simple zero at $s=1$ and analytic continuation to 
$\left\{s \in \mathbb{C}: \Re s>\frac{1}{2}\right\}$. Thus $Z_{\mathcal{P}}(s)$ has an analytic continuation to $\left\{s \in \mathbb{C}: \Re s>\frac{1}{2}\right\}$ with a simple zero at $s=1$. Thus $Z_{\mathcal{P}}(s)$ is holomorphic for $\sigma>\frac{1}{2}$ since $\zeta_{\mathcal{P}}(2 s)$ is holomorphic for $\sigma>\frac{1}{2}$. Now move the contour past the line $s=1$ to the line $\Re s=\sigma$ for any $\sigma>\frac{1}{2}$ since $Z_{\mathcal{P}}(s)$ is holomorphic in this region, we obtain

$$
I_{1}=\frac{1}{2 \pi i}\left(\int_{c-i T}^{\sigma-i T}+\int_{\sigma-i T}^{\sigma+i T}+\int_{\sigma+i T}^{c+i T}\right) \frac{Z_{\mathcal{P}}(s)}{s} x^{s} d s .
$$

These integrals will be estimated by using the bound (29). The integral over the horizontal path $[\sigma+i T, c+i T]$ is

$$
\left|\frac{1}{2 \pi i} \int_{\sigma+i T}^{c+i T} \frac{Z_{\mathcal{P}}(s)}{s} x^{s} d s\right| \ll \frac{x^{c}}{T} \exp \left\{C\left(\frac{1}{\sigma-\frac{1}{2}}\right)+C \sqrt{\frac{\log |T|}{\sigma-\frac{1}{2}}}\right\}
$$

by (29). Similarly for the integral over $[c-i T, \sigma-i T]$. On the line $\Re s=\sigma$, we will have

$$
\left|\frac{1}{2 \pi i} \int_{\sigma-i T}^{\sigma+i T} \frac{Z_{\mathcal{P}}(s)}{s} x^{s} d s\right| \ll x^{\sigma} \exp \left\{C\left(\frac{1}{\sigma-\frac{1}{2}}\right)+C \sqrt{\frac{\log |T|}{\sigma-\frac{1}{2}}}\right\} .
$$

by (29). Hence

$$
I_{1} \ll \frac{x^{c}}{T} \exp \left\{C\left(\frac{1}{\sigma-\frac{1}{2}}\right)+C \sqrt{\frac{\log |T|}{\sigma-\frac{1}{2}}}\right\}+x^{\sigma} \exp \left\{C\left(\frac{1}{\sigma-\frac{1}{2}}\right)+C \sqrt{\frac{\log |T|}{\sigma-\frac{1}{2}}}\right\},
$$

and $I_{2}$ is exactly the same as in the proof of Theorem 14 . Therefore

$$
\begin{aligned}
L_{\mathcal{P}}(x)= & I_{1}+I_{2} \ll \frac{x^{c}}{T} \exp \left\{C\left(\frac{1}{\sigma-\frac{1}{2}}\right)+C \sqrt{\frac{\log |T|}{\sigma-\frac{1}{2}}}\right\}+x^{\sigma} \exp \left\{C\left(\frac{1}{\sigma-\frac{1}{2}}\right)+C \sqrt{\frac{\log |T|}{\sigma-\frac{1}{2}}}\right\} \\
& +O\left(\frac{x^{c}}{T} \cdot \zeta_{\mathcal{P}}(c)\right)+O\left(\frac{x}{T} \sum_{\substack{\frac{x}{2}<n<2 x \\
n \in \mathcal{N}}} \frac{1}{|n-x|}\right) .
\end{aligned}
$$

Taking $c=1+\frac{1}{\log x}$ and using $\zeta_{\mathcal{P}}(1+\delta)=O\left(\frac{1}{\delta}\right)$ gives

$$
\begin{aligned}
L_{\mathcal{P}}(x) \ll & \frac{x}{T} \exp \left\{C\left(\frac{1}{\sigma-\frac{1}{2}}\right)+C \sqrt{\frac{\log |T|}{\sigma-\frac{1}{2}}}\right\}+x^{\sigma} \exp \left\{C\left(\frac{1}{\sigma-\frac{1}{2}}\right)+C \sqrt{\frac{\log |T|}{\sigma-\frac{1}{2}}}\right\} \\
& +O\left(\frac{x \log x}{T}\right)+O\left(\frac{x}{T} \sum_{\substack{x \\
\frac{x}{2}<n<2 x \\
n \in \mathcal{N}}} \frac{1}{|n-x|}\right) .
\end{aligned}
$$

By following the same argument shown in the proof of Theorem 14, we have

$$
\begin{aligned}
L_{\mathcal{P}}(x) \ll & \frac{x}{T} \exp \left\{C\left(\frac{1}{\sigma-\frac{1}{2}}\right)+C \sqrt{\frac{\log |T|}{\sigma-\frac{1}{2}}}\right\}+x^{\sigma} \exp \left\{C\left(\frac{1}{\sigma-\frac{1}{2}}\right)+C \sqrt{\frac{\log |T|}{\sigma-\frac{1}{2}}}\right\} \\
& +O\left(\frac{x \log x}{T}\right)+O\left(\frac{x^{4}}{T}\right) .
\end{aligned}
$$

Taking $T=x^{4}$, then

$$
\sum_{\substack{n \leq x \\ n \in \mathcal{N}}} \lambda_{\mathcal{P}}(n) \ll x^{\sigma} \exp \left\{C\left(\frac{1}{\sigma-\frac{1}{2}}\right)+C \sqrt{\frac{4 \log x}{\sigma-\frac{1}{2}}}\right\}
$$

since the $\mathrm{O}(1)$ are smaller than the main term. This means

$$
\sum_{\substack{n \leq x \\ n \in \mathcal{N}}} \lambda_{\mathcal{P}}(n) \ll x^{\frac{1}{2}} \exp \left\{\left(\sigma-\frac{1}{2}\right) \log x+C\left(\frac{1}{\sigma-\frac{1}{2}}\right)+C \sqrt{\frac{4 \log x}{\sigma-\frac{1}{2}}}\right\} .
$$


Now we want to minimise this quantity over $\frac{1}{2}<\sigma<2$; i.e. minimise

$$
\left(\sigma-\frac{1}{2}\right) \log x+C\left(\frac{1}{\sigma-\frac{1}{2}}\right)+C\left(\sigma-\frac{1}{2}\right)^{-\frac{1}{2}}(4 \log x)^{\frac{1}{2}}
$$

Put $\sigma=\frac{1}{2}+\frac{1}{(\log x)^{\alpha}}$ for some $\alpha>0$. This gives

$$
(\log x)^{1-\alpha}+C(\log x)^{\alpha}+C(\log x)^{\frac{1}{2}+\frac{\alpha}{2}} .
$$

This is optimal when $\alpha=\frac{1}{3}$. For $\sigma=\frac{1}{2}+\frac{1}{(\log x)^{\frac{1}{3}}}$, we have

$$
\begin{aligned}
\sum_{\substack{n \leq x \\
n \in \mathcal{N}}} \lambda_{\mathcal{P}}(n) & =O\left(x^{\frac{1}{2}} e^{C(\log x)^{\frac{1}{3}}+(1+\sqrt{4} C)(\log x)^{\frac{2}{3}}}\right) \\
& =O\left(x^{\frac{1}{2}} e^{C^{\prime \prime}(\log x)^{\frac{2}{3}}}\right) .
\end{aligned}
$$

Hence, by Abel Summation again, this gives the result.

\section{2 $\Omega$ Results for Euler's example over $\mathcal{N}$}

We now consider $\Omega$ Results of $\sum_{n \leq x} \frac{\lambda_{\mathcal{P}}(n)}{n}$ for a system $\mathcal{P}$ which satisfies either

$$
N_{\mathcal{P}}(x)=\rho x+O\left(x^{\beta}\right) \text { for some } \rho>0
$$

or

$$
\psi_{\mathcal{P}}(x)=x+O\left(x^{\alpha}\right)
$$

for some $\alpha, \beta<\frac{1}{2}$. Both of which give the lower bound $\Omega\left(\frac{1}{\sqrt{x}}\right)$ for the sum.

Proposition 18 Let $\mathcal{P}$ be a $g$-prime system satisfying (30) for some $\beta<\frac{1}{2}$. Then

$$
\sum_{\substack{n \leq x \\ n \in \mathcal{N}}} \frac{\lambda_{\mathcal{P}}(n)}{n}=\Omega\left(\frac{1}{\sqrt{x}}\right) .
$$

Proof We wish to show that (32) is true. It is enough to show that

$$
L_{\mathcal{P}}(x)=\sum_{\substack{n \leq x \\ n \in \mathcal{N}}} \lambda_{\mathcal{P}}(n)=\Omega(\sqrt{x}) .
$$

Let us assume the contrary, so that $L_{\mathcal{P}}(x)=o(\sqrt{x})$. We know that for all $\Re s>\frac{1}{2}$,

$$
Z_{\mathcal{P}}(s)=s \int_{1}^{\infty} \frac{L_{\mathcal{P}}(x)}{x^{s+1}} d x
$$

is holomorphic. But for $\Re_{i}>1,\left(Z_{\mathcal{P}}(s)=\right) \frac{\zeta_{\mathcal{P}}(2 s)}{\zeta_{\mathcal{P}}(s)}$ and $\zeta_{\mathcal{P}}(2 s)$ is holomorphic for $\Re s>\frac{1}{2}$ with no zeros for $\Re_{s}>\frac{1}{2}$ since $\zeta_{\mathcal{P}}(s)$ is holomorphic for $\Re_{S}>1$ and has no zeros here. For $\Re s>1$, we have $\zeta_{\mathcal{P}}(s)=\frac{\zeta_{\mathcal{P}}(2 s)}{Z_{\mathcal{P}}(s)}$ which has a meromorphic continuation for $\Re s>\frac{1}{2}$ except for a pole at $s=1$ and no zeros. Therefore $\zeta_{\mathcal{P}}(2 s)$ has a meromorphic continuation for $\Re s>\frac{1}{4}$ and pole at $s=\frac{1}{2}$. Now we know from the assumption (30) that $\zeta_{\mathcal{P}}(s)$ has an analytic continuation for $\sigma>\beta$ except for a simple pole at $s=1$. Thus $Z_{\mathcal{P}}(s)$ is 
holomorphic for $\sigma=\Re s>\frac{1}{2}$ with pole at $s=\frac{1}{2}$. On the one hand, we know that $Z_{\mathcal{P}}(s)$ has a pole at $\frac{1}{2}$ since $\zeta_{\mathcal{P}}(2 s)$ has a simple pole at $\frac{1}{2}$. Thus

$$
Z_{\mathcal{P}}\left(\frac{1}{2}+\epsilon\right) \sim \frac{C}{\epsilon^{k}} \text { for some } k \geq 1 \text { and } C \neq 0 .
$$

On the other hand, from the right integral of (33) with $s=\frac{1}{2}+\epsilon$, we have

$$
\left|Z_{\mathcal{P}}\left(\frac{1}{2}+\epsilon\right)\right|=\left|\left(\frac{1}{2}+\epsilon\right) \int_{1}^{\infty} \frac{L_{\mathcal{P}}(x)}{x^{\frac{3}{2}+\epsilon}} d x\right| \leq\left|\frac{1}{2}+\epsilon\right| \int_{1}^{\infty} \frac{\left|L_{\mathcal{P}}(x)\right|}{x^{\frac{3}{2}+\epsilon}} d x
$$

$\left|L_{\mathcal{P}}(x)\right|$ can be written as $g(x) \sqrt{x}$, where $g(x) \geq 0$ and $g(x) \longrightarrow 0$ as $x \longrightarrow \infty$. Hence we have

$$
\left|Z_{\mathcal{P}}\left(\frac{1}{2}+\epsilon\right)\right| \leq B \int_{1}^{\infty} \frac{g(x) \sqrt{x}}{x^{\frac{3}{2}+\epsilon}} d x=B \int_{1}^{\infty} \frac{g(x)}{x^{1+\epsilon}} d x \text {, where } B \text { is constant. }
$$

Clearly

$$
\int_{1}^{\infty} \frac{g(x)}{x^{1+\epsilon}} d x=o\left(\frac{1}{\epsilon}\right)
$$

This also implies that

$$
\int_{1}^{\infty} \frac{L_{\mathcal{P}}(x)}{x^{\frac{3}{2}+\epsilon}} d x=o\left(\frac{1}{\epsilon}\right)
$$

so that $Z_{\mathcal{P}}\left(\frac{1}{2}+\epsilon\right)=o\left(\frac{1}{\epsilon}\right)$. Therefore this gives a contradiction with (34) and hence

$$
\sum_{\substack{n \leq x \\ n \in \mathcal{N}}} \frac{\lambda_{\mathcal{P}}(n)}{n}=\Omega\left(\frac{1}{\sqrt{x}}\right) .
$$

In order to show (31) implies (32), we first need to prove a more general result.

Proposition 19 If $\mathcal{P}$ is a g-prime system for which

$$
N_{\mathcal{P}}(x) \sim \rho x \text { for some } \rho>0,
$$

and $\zeta_{\mathcal{P}}(s)$ has an analytic continuation past $\Re$ s $=1$ to a region containing a neighborhood of $s=\frac{1}{2}$, then (32) holds.

Proof To show (32) is true, it is enough to show that

$$
L_{\mathcal{P}}(x)=\sum_{\substack{n \leq x \\ n \in \mathcal{N}}} \lambda_{\mathcal{P}}(n)=\Omega(\sqrt{x}) .
$$

Let us again assume that $L_{\mathcal{P}}(x)=o(\sqrt{x})$. We know that

$$
Z_{\mathcal{P}}(s)=s \int_{1}^{\infty} \frac{L_{\mathcal{P}}(x)}{x^{s+1}} d x
$$

is holomorphic for all $\Re s>\frac{1}{2}$. But for $\Re s>1,\left(Z_{\mathcal{P}}(s)=\right) \frac{\zeta \mathcal{P}(2 s)}{\zeta_{\mathcal{P}}(s)}$ and $\zeta_{\mathcal{P}}(2 s)$ is holomorphic for $\Re s>\frac{1}{2}$ with no zeros for $\Re>\frac{1}{2}$ since $\zeta_{\mathcal{P}}(s)$ is holomorphic for $\Re s>1$ and has no zeros here. For $\Re s>1$, we have $\zeta_{\mathcal{P}}(s)=\frac{\zeta_{\mathcal{P}}(2 s)}{Z_{\mathcal{P}}(s)}$ which has a meromorphic continuation for $\Re s>\frac{1}{2}$ except for a pole at $s=1$ and no zeros. Thus $\zeta_{\mathcal{P}}(2 s)$ has a meromorphic continuation for $\Re s>\frac{1}{4}$ and pole at $s=\frac{1}{2}$. Thus $Z_{\mathcal{P}}(s)$ is holomorphic for $\sigma=\Re s>\frac{1}{2}$ with pole at $s=\frac{1}{2}$ because $\zeta_{\mathcal{P}}(s)$ is holomorphic at $\frac{1}{2}$.

Hence, by following the same argument shown in the proof of Proposition 18, we obtain the required result. 
Remark 20 With Zhang's system which was previously detailed in the paper, then (32) is also true if we assume $\zeta_{\mathcal{P}}(s)$ has an analytic continuation to a neighborhood of $s=\frac{1}{2}$.

In the following corollary we consider the effect of the assumption (31) for some $\alpha<\frac{1}{2}$.

Corollary 21 Let $\mathcal{P}$ be a g-prime system satisfying (31) for some $\alpha<\frac{1}{2}$. Then (32) holds.

Proof By Theorems 2.1 and 2.2 of [13], the assumption (31) for some $\alpha<\frac{1}{2}$ implies $\zeta_{\mathcal{P}}(s)$ is holomorphic for $\Re s>\alpha$ except for a simple pole at $s=1$ and that it has no zeros in this region, and

$$
N_{\mathcal{P}}(x)=\rho x+O\left(x^{-c \sqrt{\log x \log \log x}}\right)
$$

for some $\rho>0$ (see [13]). Hence, by Proposition 19, (32) holds.

Remark 22 Let $\mathcal{P}$ be a $g$-prime system satisfying (19) and (20) for some $\alpha, \beta,<1$ respectively and define $\xi$ via

$$
L_{\mathcal{P}}(x)=\sum_{\substack{n \leq x \\ n \in \mathcal{N}}} \lambda_{\mathcal{P}}(n)=O\left(x^{\xi+\varepsilon}\right)
$$

holds for all $\varepsilon>0$, but for no $\varepsilon<0$ and for some $\xi<1$. Then Proposition 18 and Corollary 21 imply that $\max \{\beta, \xi\} \geq \frac{1}{2}$ and $\max \{\alpha, \xi\} \geq \frac{1}{2}$.

\section{Open problem}

As mentioned in earlier, Kahane and Saïas proposed that for all CMO functions, one has $\sum_{n \leq x} f(n)=\Omega\left(\frac{1}{\sqrt{x}}\right)$. They also showed that GRH-RH (Generalised Riemann Hypothesis-Riemann Hypothesis) would follow from this result. In our findings, we did not find any $C M O_{\mathcal{P}}$ functions $f$ such that $\sum_{n \leq x} f(n)=O\left(\frac{1}{x^{c}}\right)$ for $c>\frac{1}{2}$. This may suggest the following conjecture.

Conjecture 23 Let $\mathcal{P}$ be a $g$-prime system with abscissa 1 . Then, for all completely multiplicative functions on $\mathcal{N}$, we have

$$
\sum_{\substack{n \leq x \\ n \in \mathcal{N}}} f(n)=\Omega\left(\frac{1}{\sqrt{x}}\right) .
$$

Received: 22 May 2020 Accepted: 13 September 2020 Published online: 28 October 2020

\section{References}

1. Balazard, M., Roton, A.D.: Notes de lecture de l'article Partial sums of the Mobius function de Kannan Soundararajan (2008). arXiv preprint arXiv:0810.3587

2. Bateman, P.T., Diamond, H.G.: Asymptotic distribution of Beurling's generalized prime numbers. Stud. Number Theory 6, 152-212 (1969)

3. Beurling, A.: Analyse de la loi asymptotique de la distribution des nombres premiers généralisés. I. Acta Math. 68 255-291 (1937)

4. Broucke, F., Debruyne, G., Vindas, J.: Beurling integers with RH and large oscillation. Adv. Math. 370, 107240 (2020)

5. Debruyne, G., Maes, F., Vindas, J.: Halász's theorem for Beurling generalized numbers. Acta Arith. 194, 59-72 (2020)

6. Debruyne, G., Vindas, J.: On PNT equivalences for Beurling numbers. Mon. Math. 184(3), $401-424$ (2017)

7. Debruyne, G., Vindas, J.: On Diamond's L1 criterion for asymptotic density of Beurling generalized integers. Mich. Math. J. 68(1), 211-223 (2019)

8. Diamond, H.G.: A set of generalized numbers showing Beurling's theorem to be sharp. III. J. Math. 14(1), 29-34 (1970) 
9. Diamond, H.G., Zhang, W.-B.: A PNT equivalence for Beurling numbers. Funct. Approx. Comment. Math. 46(2), 225-234 (2012)

10. Diamond, H.G., Zhang, W.-B.: Beurling Generalized Numbers. American Mathematical Society, Providence (2016)

11. Hilberdink, T.W.: Well-behaved Beurling primes and integers. J. Number Theory 112, 332-344 (2005)

12. Hilberdink, T.W.: Generalised prime systems with periodic integer counting function. Acta Arith. 152(3), 217-241 (2012)

13. Hilberdink, T.W., Lapidus, M.L.: Beurling zeta functions, generalised primes, and fractal membranes. Acta Appl. Math. 94, 21-48 (2006)

14. Kahane, J.-P., Saïas, E.: Fonctions complétement multiplicatives de somme nulle. Expo. Math. 35(4), 364-389 (2017)

15. Neamah, A.A., Hilberdink, T.W.: The average order of the Mobius function for Beurling prime. Int. J. Number Theory 16(5), 1005-1011 (2020)

16. Zhang, W.-B.: A generalization of Halász's theorem to Beurling's generalized integers and its application. III. J. Math. 31(4), 645-664 (1987)

17. Zhang, W.-B.: Beurling primes with RH, Beurling primes with large oscillation. Math. Ann. 337, 671-704 (2007)

18. Zhang, W.-B.: Halász's theorem for Beurling numbers. Acta Arith. 183, 223-235 (2018)

\section{Publisher's Note}

Springer Nature remains neutral with regard to jurisdictional claims in published maps and institutional affiliations. 